\title{
A STEINHAUS TYPE THEOREM
}

\author{
P. N. NATARAJAN
}

ABStRACT. The sequence space $\Lambda_{r}, r \geq 1$ being a fixed integer, is defined as

$$
\Lambda_{r}=\left\{x=\left\{x_{k}\right\} \in l_{\infty}, x_{k} \in K, k=0,1,2, \ldots,\left|x_{k+r}-x_{k}\right| \rightarrow 0, k \rightarrow \infty\right\},
$$

where $K$ is a complete, nontrivially valued field and $l_{\infty}$ is the space of bounded sequences with entries in $K$. In this paper, it is proved that given a regular matrix $A=\left(a_{n k}\right), a_{n k} \in K=\mathbf{R}$ or $\mathbf{C}$, there exists a sequence in $\Lambda_{r}-\bigcup_{i=1}^{r-1} \Lambda_{i}$ which is not $A$-summable. This is an improvement of the well-known Steinhaus theorem. It is, however, shown that this result fails to hold when $K$ is a complete, nontrivially valued, nonarchimedean field, whereas it is known that the Steinhaus theorem continues to hold.

In this paper, $K$ denotes $\mathbf{R}$, the field of real numbers or $\mathbf{C}$, the field of complex numbers, or a complete, nontrivially valued, nonarchimedean field. In the relevant context, we explicitly mention which field is chosen.

If $A=\left(a_{n k}\right), a_{n k} \in K, n, k=0,1,2, \ldots,{ }^{\prime}$ is an infinite matrix and $x=\left\{x_{k}\right\}, x_{k} \in$ $K, k=0,1,2, \ldots$, by the $A$-transform of $x$, we mean the sequence $\left\{(A x)_{n}\right\}$ where

$$
(A x)_{n}=\sum_{k=0}^{\infty} a_{n k} x_{k}, \quad n=0,1,2, \ldots,
$$

assuming that the series on the right converge. $x$ is said to be summable by the matrix method $A$ or $A$-summable to $s$ if

$$
(A x)_{n} \rightarrow s, \quad \text { as } n \rightarrow \infty .
$$

If $X, Y$ are sequence spaces with elements whose entries are in $K$ and if $A=\left(a_{n k}\right)$, $a_{n k} \in K, n, k=0,1,2, \ldots$, is an infinite matrix, $A$ is said to transform $X$ to $Y$, written as $A \in(X, Y)$, if whenever $x=\left\{x_{k}\right\} \in X,(A x)_{n}$ is defined, $n=0,1,2, \ldots$, and $\left\{(A x)_{n}\right\} \in Y$.

$l_{\infty}$ denotes the set of all bounded sequences with entries in $K$. For $x=\left\{x_{k}\right\} \in$ $l_{\infty}$, the norm of $x$ is defined by

$$
\|x\|=\sup _{k \geq 0}\left|x_{k}\right|
$$

$l_{\infty}$ is seen to be a Banach space if $K=\mathbf{R}$ or $\mathbf{C}$ and a nonarchimedean Banach space (see e.g. [2] for the definition) if $K$ is a complete, nontrivially valued, nonarchimedean field. $c$ denotes the set of all convergent sequences with entries in $K$. With respect to the norm defined by (1), $c$ is a closed subspace of $l_{\infty}$.

If $A=\left(a_{n k}\right), a_{n k} \in K, n, k=0,1,2, \ldots, A \in(c, c)$, and $\lim _{n \rightarrow \infty}(A x)_{n}=$ $\lim _{k \rightarrow \infty} x_{k}, A$ is called a regular or Toeplitz matrix and we write $A \in(c, c ; P)$. $A$

Received by the editors July 2, 1985 and, in revised form, January 2, 1986.

1980 Mathematics Subject Classification (1985 Revision). Primary 40C05; Secondary 40D25. 
is called a Schur matrix if $A \in\left(l_{\infty}, c\right)$. The well-known Steinhaus theorem can now be symbolically written as

$$
(c, c ; P) \cap\left(l_{\infty}, c\right)=\varnothing
$$

In this paper, the space $\Lambda_{r} \subset l_{\infty}$ (see [3]) is studied in the context of the Steinhaus theorem. It may be recalled that the space $\Lambda_{r}, r \geq 1$ being a fixed integer, is the set of all $\left\{x_{k}\right\} \in l_{\infty}$ such that $\left|x_{k+r}-x_{k}\right| \rightarrow 0$, as $k \rightarrow \infty$. $\Lambda_{r}$ is a closed subspace of $l_{\infty}$ with respect to the norm defined by (1).

For results such as the Steinhaus theorem mentioned above, a reference would be [4].

The following result, improving the Steinhaus theorem, is proved here.

THEOREM 1. $(c, c ; P) \cap\left(\Lambda_{r}-\bigcap_{i=1}^{r-1} \Lambda_{i}, c\right)=\varnothing$ when $K=\mathbf{R}$ or $\mathbf{C}$.

ProOF. Let $A=\left(a_{n k}\right)$ be a regular matrix. Then we can choose two sequences of positive integers $\{n(m)\},\{k(m)\}$, such that if

$$
m=2 p, \quad n(m)>n(m-1), \quad k(m)>k(m-1)+(2 m-5) r
$$

then

$$
\begin{gathered}
\sum_{k=0}^{k(m-1)+(2 m-5) r}\left|a_{n(m), k}\right|<\frac{1}{16}, \\
\sum_{k=k(m)+1}^{\infty}\left|a_{n(m), k}\right|<\frac{1}{16}
\end{gathered}
$$

and if

$$
m=2 p+1, \quad n(m)>n(m-1), \quad k(m)>k(m-1)+(m-2) r
$$

then

$$
\begin{gathered}
\sum_{k=0}^{k(m-1)+(m-2) r}\left|a_{n(m), k}\right|<\frac{1}{16} \\
\sum_{k=k(m-1)+(m-2) r+1}^{k(m)}\left|a_{n(m), k}\right|>\frac{7}{8} \\
\sum_{k=k(m)+1}^{\infty}\left|a_{n(m), k}\right|<\frac{1}{16} .
\end{gathered}
$$


Define the sequence $x=\left\{x_{k}\right\}$ as follows: if $k(2 p-1)<k \leq k(2 p)$, then

$$
x_{k}=\left[\begin{array}{cc}
\frac{2 p-2}{2 p-1}, & k=k(2 p-1)+1, \\
1, & k(2 p-1)+1<k \leq k(2 p-1)+r, \\
\frac{2 p-3}{2 p-1}, & k=k(2 p-1)+r+1, \\
1, & k(2 p-1)+r+1<k \leq k(2 p-1)+2 r, \\
\vdots & k(2 p-1)+(2 p-4) r+1<k \\
1, & \leq k(2 p-1)+(2 p-3) r \\
\frac{1}{2 p-1}, & k=k(2 p-1)+(2 p-3) r+1, \\
\frac{2 p-2}{2 p-1}, & k(2 p-1)+(2 p-3) r+1<k \\
0, & \leq k(2 p-1)+(2 p-2) r \\
\frac{2 p-3}{2 p-1}, & k(2 p-1)+(2 p-2) r+1<k \\
0, & k=k(2 p-1)+(2 p-1) r, \\
\vdots & \\
\frac{1}{2 p-1}, & k(2 p-1)+(4 p-6) r+1<k \\
0, & k(2 p-1)+(4 p-5) r<k \leq k(2 p),
\end{array}\right.
$$

and if $k(2 p)<k \leq k(2 p+1)$, then

$$
x_{k}=\left[\begin{array}{cl}
\frac{1}{2 p}, & k(2 p)<k \leq k(2 p)+r, \\
\frac{2}{2 p}, & k(2 p)+r<k \leq k(2 p)+2 r, \\
\vdots & \\
\frac{2 p-1}{2 p}, & k(2 p)+(2 p-2) r<k \leq k(2 p)+(2 p-1) r, \\
1, & k(2 p)+(2 p-1) r<k \leq k(2 p+1) .
\end{array}\right.
$$

We note that, if $k(2 p-1)<k \leq k(2 p)$,

$$
\left|x_{k+r}-x_{k}\right|<\frac{1}{2 p-1}
$$

while, if $k(2 p)<k \leq k(2 p+1)$,

$$
\left|x_{k+r}-x_{k}\right|<\frac{1}{2 p}
$$

Thus $\left|x_{k+r}-x_{k}\right| \rightarrow 0$, as $k \rightarrow \infty$, showing that $x=\left\{x_{k}\right\} \in \Lambda_{r}$. However,

$$
\left|x_{k+1}-x_{k}\right|=\frac{2 p-2}{2 p-1} \quad \text { if } k=k(2 p-1)+(2 p-3) r, p=1,2, \ldots
$$


Hence $\left|x_{k+1}-x_{k}\right| \nrightarrow 0$, as $k \rightarrow \infty$, and consequently $x \notin \Lambda_{1}$. In a similar manner, we can prove that $x \notin \Lambda_{i}, i=2,3, \ldots, r-1$. Thus $x \in \Lambda_{r}-\bigcup_{i=1}^{r-1} \Lambda_{i}$. Further

$$
\left.\begin{array}{l}
\left|(A x)_{n(2 p)}\right|<\frac{1}{16}+\frac{1}{16}=\frac{1}{8}, \\
\left|(A x)_{n(2 p+1)}\right|>\frac{7}{8}-\frac{1}{16}-\frac{1}{16}=\frac{3}{4}
\end{array}\right\}, \quad p=1,2, \ldots,
$$

which shows that $\left\{(A x)_{n}\right\} \notin c$. This completes the proof of the theorem.

REMARK. In the context of Theorem 1, one may enquire whether a matrix which sums all sequences of 0 's and 1's in $\bigcup_{r=1}^{\infty} \Lambda_{r}$ sums all sequences in $\bigcup_{r=1}^{\infty} \Lambda_{r}$. The answer to this query is, however, in the negative. For, if $x=\left\{x_{k}\right\}$ is a sequence of 0 's and 1's in $\bigcup_{r=1}^{\infty} \Lambda_{r}$, then $x_{k+r}=x_{k}$ for $k \geq K_{0}$ and for some integer $r \geq 1$, i.e., $x$ is eventually periodic and hence summable $(C, 1)$ as is seen directly or using the idea of almost convergence (see [4, p. 12]). The sequence

$$
\left\{0 ; 1,0 ; \frac{1}{2}, \frac{2}{2}, \frac{1}{2}, 0 ; \frac{1}{4}, \frac{2}{4}, \frac{3}{4}, \frac{4}{4}, \frac{3}{4}, \frac{2}{4}, \frac{1}{4}, 0 ; \ldots\right\}
$$

is in $\Lambda_{1}$ but it is not summable $(C, 1)$.

In the context of Theorem 1, Theorem 2 below indicates a deviation from the classical case in the nonarchimedean case.

THEOREM 2. There exists a regular matrix with entries in $Q_{p}$, the p-adic field for a prime $p$, which sums all sequences in $\Lambda_{r}$ for a fixed positive integer r, i.e. $(c, c ; P) \cap\left(\Lambda_{r}, c\right) \neq \varnothing$.

ProOF. Consider the matrix $A=\left(a_{n k}\right), a_{n k} \in Q_{p}, n, k=0,1,2, \ldots$, where

$$
\begin{aligned}
& \left.\begin{array}{rlrl}
a_{n k} & =\frac{1}{r}, & & k=n, n+1, \ldots, n+r-1 \\
& =0 & & \text { otherwise }
\end{array}\right\}, \quad n=0,1,2, \ldots . \\
& (A x)_{n+1}-(A x)_{n}=\frac{\left(x_{n+1}+x_{n+2}+\cdots+x_{n+r}\right)-\left(x_{n}+x_{n+1}+\cdots+x_{n+r-1}\right)}{r} \\
& =\frac{x_{n+r}-x_{n}}{r} \rightarrow 0, \quad \text { as } n \rightarrow \infty,
\end{aligned}
$$

if $x=\left\{x_{k}\right\} \in \Lambda_{r}$. Then $A$ sums all sequences in $\Lambda_{r}$. It is clear that $A$ is regular.

I thank the referee for putting the remark preceding Theorem 2 in the proper perspective.

\section{REFERENCES}

1. G. Bachman, Introduction to $p$-adic numbers and valuation theory, Academic Press, New York, 1964.

2. L. Narici, E. Beckenstein, and G. Bachman, Functional analysis and valuation theory, Dekker, New York, 1971.

3. P. N. Natarajan, The Steinhaus theorem for Toeplitz matrices in non-archimedean fields, Comment. Math. Prace Mat. 20 (1978), 417-422.

4. K. Zeller and W. Beekmann, Theorie der Limitierungsverfahren, Springer, Berlin and New York, 1970.

Department of Mathematics, Vivekananda College, Madras-600 004, India 